\title{
Meditation, mindfulness, and critical thinking: individual characteristics in online higher education.
}

\author{
Alicia Holland*, Gwendolyn Dooley, Barbara Fedock, Susan Ferebee, Liston Bailey \\ School of Advanced Studies, University of Phoenix, Phoenix, USA
}

\begin{abstract}
Limited research has been conducted on the relationship between age, ethnicity and gender and the use of meditation to strengthen critical thinking. The purpose of this research study will be to investigate the relationship of age, gender and ethnicity to the use of meditation to improve critical thinking. As a quantitative study the researchers sought to quantify the strength of relationships across these variables while making predictive judgments about the potential value of mindfulness approaches on learning. Participants in this study were members of a purposive sample of twenty-two (22) students attending a Holistic Healing Arts school in the western part of the United States. The dependent variable being considered is the improvement of critical thinking. The independent variables discussed in this study are factors related to age, ethnicity, gender and the use of mindfulness methods. The critical thinking skills were measured by the California Critical Thinking Skills Test-Numeracy (CCTST-N) validated/reliable online instrument. The Mindful Attention Awareness Scale (MAAS) was used to measure mindfulness.
\end{abstract}

Keywords: Meditation, Mindfulness, Critical thinking, Online, Higher education

Accepted on July 17, 2017

\section{Introduction}

There is a need for research to focus on meditation in educational settings for youths and other age ranges [1]. Several studies show that mindfulness results in enhanced self-regulation across a spectrum of behaviors such as procrastination [2], the ability to persist [3], alcohol reduction [4], and improved eating habits [5]. Mindfulness is often described as a contemplative practice that allows one to develop concentration, to deepen understanding and insight, and to cultivate awareness and compassion [6]. When mindfulness is viewed through an educational lens, there appears to be potentiality for its use within the learning environment in classrooms, both online and in face to face settings. Some have described mindfulness as a desirable condition within the educational environment that promotes creativity and enhances transfer of skills and knowledge to new contexts [7]. A more quixotic interpretation of mindfulness is its utility as a tool to attenuate risks to our well-being that result from an overload of information transmission and the stressful events we encounter on a daily basis [8]. In practice, it is believed that through developed skill at using techniques such as focused attention and open monitoring, people can reach mindfulness and meditation goals [9]. For this study, it was difficult to find specific literature using the keywords of critical thinking, meditation, and gender. Therefore, the current research may begin further exploration on the relationship of gender to mediation and critical thinking in higher education.

\section{Significance of the Problem}

There is limited research on critical thinking regarding secondary and higher education students' ethnicity and the use of meditation. However, the academic and critical thinking benefits of meditation are evident in the research [10]. One significance within the literature is that secondary and higher education students from all ethnic groups tend to exhibit higher critical thinking skills after engaging in meditation [11]. There appears to be a sense that a complex relationship across these multiple factors that might affect levels of improved critical thinking in individuals. One recent study pointed to the possibility that executive function (EF) may have a mediator effect regarding the relationship between mindfulness meditation and critical thinking (CT) [12]. However, this study did not lament that the true mechanisms by which EF supports CT have not yet been established, nor are they fully understood.

Students may consider learning the skill of mindfulness meditation. Mindfulness-based interventions with a focus on teaching mindfulness skills to promote psychological health and well-being are well represented and prominent in current literature. However, the mindfulness interventions presented by researchers include a focus on pain management but not critical thinking mindfulness and higher education. There is literature on mindfulness based interventions that focuses on teaching mindfulness skills to promote psychological health and well-being. However, mindfulness interventions aligned with individual characteristics related to age, ethnicity, gender on the improvement of critical thinking skills using meditation could be of value to educational practitioners and psychologists. Many of these mindfulness interventions are 
in pain management but not critical thinking. However, there is a need for research to focus on meditation in educational settings for youths and other age ranges [1]. Perhaps the findings of this study could show additional data on educational practices.

\section{Literature Review}

Within this study, mindfulness meditation is explored for indicators of how its practice may impact critical thinking among diverse populations. Specifically, the purpose of this study is to examine the relationship of the unknown effects of individual characteristics related to age, ethnicity, gender and the improvement of critical thinking skills using meditation. The independent variables are age, ethnicity, gender. The dependent variable is improvement of critical thinking skills using meditation. Meditation practice is viewed as an added benefit of improved physical and mental health because the individual may achieve a level of mental processing devoid of stress, negative thoughts, or conflicting emotions. Popular themes in current research surround exploring effects of mindfulness meditation on aspects mental resilience and cognitive functioning. For example, a study using a pretestposttest design $(n=22)$ completed by Mracek et al. suggested that cultivating mindfulness was an effective technique for improving working memory and cognitive functioning among students preparing to take the GRE exam [13]. But few studies have fully discerned relationships between mindfulness, executive functioning (EF) and critical thinking (CT). Additionally, limited research presented findings on effects of individual characteristics related to age, ethnicity, gender on the improvement of critical thinking skills using meditation. The themes of this study focus on mindfulness, meditation and critical thinking.

\section{Background of the Problem}

There is a need for research to focus on meditation in educational settings for youths and other age ranges [1]. Several studies show that mindfulness results in enhanced self-regulation across a spectrum of behaviors such as procrastination [2], the ability to persist [3], alcohol reduction [4] and improved eating habits [5]. Burton et al. demonstrated that executive function process is related to inhibition [14]. Updating support for higher-order thought in decisionmaking, solving problems, and metacognition suggests that executive function is necessary for new scenarios where there is not an automatic previously learned response.

\section{Mindfulness Factors and their Cognitive Effects}

Mindfulness, defined as "clarity of thought," stems from Buddhist traditions [12,15]. While therapist and researchers appear to support the use of mindfulness during psychotherapy sessions to increase self-compassion [16], there is emerging research to suggests benefits for higher education. Noone et al. reported three primary views for critical thinking and mindfulness [12].
- No relationship exists between mindfulness and critical thinking;

- Mindfulness may be a barrier for critical thinking skills because of an individual's acceptance or nonreactive processing;

- Mindfulness may influence effective CT because of improved self-regulation.

While Noone et al. [12] provided these benefits for higher education integrating mindfulness and critical in curricula, Kiener et al. [17] found that some university programs contain classes that cause students to use lower level thinking such as memorization, thus insufficiently meeting the demands of a 21st century workforce. Educators and administrators also have limited time to achieve program outcomes and risk job elimination if these $21^{\text {st }}$ century demands are unmet [15]. There is an ongoing line of inquiry in the educational literature on the utility of mindfulness meditation training for counselor education. Others suggested that mindfulness can benefit counselor and health care professionals looking for ways to improve the experiences of those with whom they counsel or consult [18]. Fulton and Cashwell suggested that mindfulness training can be added to counselor training to address factors related to empathy and anxiety within the counselor role [19].

\section{Mindfulness and Critical Thinking}

Within a review of literature describing the merits of mindfulness, one finds numerous articles on mind-body experiences that allow individuals to think better or cope with pain and emotions. Mindfulness is often described as a contemplative practice that allows one to develop concentration, to deepen understanding and insight, and to cultivate awareness and compassion [6]. When mindfulness is viewed through an educational lens, there appears to be potentiality for its use within the learning environment in classrooms, both online and in face to face settings. Some have described mindfulness as a desirable condition within the educational environment that promotes creativity and enhances transfer of skills and knowledge to new contexts [7]. A more quixotic interpretation of mindfulness is its utility as a tool to attenuate risks to our well-being that result from an overload of information transmission and the stressful events we encounter on a daily basis [8]. In practice, it is believed that through developed skill at using techniques such as focused attention and open monitoring, people can reach mindfulness and meditation goals [9].

\section{Mindfulness and Education Levels}

Numerous articles in related educational writings point to perceived benefits of meditation and contemplative practices for different levels of students [1,6,20]. However, few of these articles investigate linkages between critical thinking and mindfulness practices among students at different levels. Also, few formal experimental studies reveal predictive data related to the probability that using mindfulness techniques 
allow students to gain academically. However, there have been a few researchers, who suggest mindfulness can promote better thinking. For example, in one particular article, it was asserted that mindfulness meditation supported better executive functioning in individuals and therefore supported optimized learning potential [21]. The idea is that by teaching learners to control their attention and concentration levels, better learning is likely to result.

For youth and adolescents' mindfulness meditation training can be described as an avenue for developing regulatory skills that will help them to cope with challenges faced as they grow and develop into adults. Some researchers have looked at mindfulness training as a way to develop prosocial behavior, emotional regulation and coping skills among youth [1]. At the primary school level, there are beliefs among educators that mindfulness approaches may benefit students' educators increasingly believe that mindfulness can be used to address mental health challenges that kids face such as anxiety, trouble paying attention and bullying [22].

Black and Fernando previously conducted a study of youth in an elementary school setting in which teachers reported better behavior patterns after students were exposed to mindfulness training [23]. Within this study variable related to paying attention, self-control, participation, and respect for others was assessment with the data showing positive trends. Viafora et al. [24] found in a small quasi-experimental study that middle school students benefitted from mindfulness training because it served as a mechanism for coping with emotions related to stress and anger. Wisner found indicators to support the idea that when adolescents were engaged in 10-12 min of school-based meditation practices several times a week, the result was a less stressful learning environment [25].

\section{Mindfulness Utility for College Students}

For college students, mindfulness and meditation training may have increasing value for helping them to deal with adjustment difficulties while attending the university [26]. College students may derive physiological benefits from mindfulness and meditation activities related to decreased test anxiety, nervousness, self-doubt and concentration loss. Having the ability to engage in contemplative states (for e.g. prayer, meditation or mindfulness) may support the mental health of individuals to the extent that they become more positive about the world around them [27].

\section{Gender Using Meditation to Strengthen Critical Thinking}

For this study, it was difficult to find specific literature using the keywords of critical thinking, meditation, and gender. Therefore, the current research may begin further exploration on the relationship of gender to mediation and critical thinking in higher education.

\section{Conceptual Framework}

The critical thinking theoretical framework [28] is a three- pronged approach in which a person controls the thinking process by acting on essential principles of thinking and creating a scholarly operational framework. Per Sergeant and Camion, the foundation of the critical thinking framework is based on Bloom's taxonomy of learning [29]. The tenets originate from 6 sequential levels: (a) knowledge, (b) comprehension, (c) application, (d) analysis, (e) synthesis, and (f) evaluation. The elements in the Paul-Elder framework are (a) elements of thought, (b) intellectual standards, and (c) intellectual traits. Higher education instructors may expect students to be responsible for developing and transferring critical thinking skills from situation to situation and course to course. However, students may not be skilled to take control of the learning process, or they may lack training in the use of mindfulness approaches to support dimensions of critical thinking.

For this study, the critical thinking framework grounds the study. According to researchers, there is difficulty in separating student views regarding learning and institutional practices. The researchers examined the potential connections between critical thinking and the use of mindfulness approaches among individuals from diverse populations and demographic data. The researchers determined that it would be of interests to curriculum developers and teachers to understand how diverse populations may engage critical thinking through mindfulness. Several studies show that mindfulness results in enhanced self-regulation across a spectrum of behaviors such as procrastination [2], the ability to persist [3], alcohol reduction [4] and improved eating habits [5]. Burton et al. demonstrated that executive function process is related to inhibition, and updating support higher-order thought in decision-making, solving problems and metacognition and suggest that executive function is necessary for new scenarios where there is not an automatic previously learned response and additional control is needed [14]. Executive function, therefore, might be necessary to support efforts to apply critical thinking and based on Chiesa et al.'s work, mindfulness may support executive function and executive function intercedes between affect (positive and negative) and mindfulness [30,31].

\section{Purpose of the Study}

The purpose of this study is to examine the relationship of the unknown effects of individual characteristics related to age, ethnicity, gender and the improvement of critical thinking skills using meditation. The independent variables are age, ethnicity, gender. The dependent variable is improvement of critical thinking skills using meditation. Meditation practice is viewed as an added benefit of improved physical and mental health because the individual may achieve a level of mental processing devoid of stress, negative thoughts or conflicting emotions. Popular themes in current research surround exploring effects of mindfulness meditation on aspects mental resilience and cognitive functioning. For example, a study using a pretest-posttest design $(\mathrm{n}=22)$ completed by Mracek et al. suggested that cultivating mindfulness was an effective 
technique for improving working memory and cognitive functioning among students preparing to take the GRE exam [13]. But few studies have fully discerned relationships between mindfulness, executive functioning $(\mathrm{EF})$ and critical thinking (CT). Additionally, limited research presented findings on effects of individual characteristics related to age, ethnicity and gender on the improvement of critical thinking skills using meditation. The themes of this study focus on mindfulness, meditation and critical thinking.

\section{Research Questions/Hypotheses}

In this causal comparative study, the overarching research question is what is the relationship of the unknown effects of individual characteristics related to age, ethnicity, gender on the improvement of critical thinking skills using meditation. The independent variables are age, ethnicity, gender. The dependent variable is improvement of critical thinking skills using meditation. The sub research questions and null and alternate hypotheses follow below:

(1) What will be the significant statistical difference based on the effect of age on improvement of critical thinking skills as a result of using meditation?

$H_{0 I}$ : A significant statistical difference will not exist based on the effect of age [d3] on improvement of critical thinking skills using meditation.

$H_{A I}:$ A significant statistical difference will exist based on the effect of age on improvement of critical thinking skills using meditation.

(2) What will be the significant statistical difference based on the effect of ethnicity on improvement of critical thinking skills using meditation?

$H_{02}$ : A significant statistical difference will not exist based on the effect of ethnicity on improvement of critical thinking skills using meditation.

$H_{A 2}$ : A significant statistical difference will exist based on the effect of ethnicity on improvement of critical thinking skills using meditation.

(3) What will be the significant statistical difference based on the effect of gender on improvement of critical thinking skills using meditation?

$H_{13}$ : A significant statistical difference will not exist based on the effect of gender on improvement of critical thinking skills using meditation.

$H_{A 3}$ : A significant statistical difference will exist based on the effect of gender on improvement of critical thinking skills using meditation.

\section{Methods and Materials}

\section{Participants}

Twenty-two participants undergraduate students (Mean age; 39.6; 19 females; 3 males) attending the Southwest Institute of Healing Arts of Phoenix, Arizona participated in the study. A purposive sampling approach was used to select the sample. Participants were recruited by emails sent to students who were already enrolled in entry level meditation courses offered by the institution that lasted five weeks. In the first week of the course, participants consented to participant in the study and completed both the mindfulness survey and pre-test of the critical thinking instrument. Throughout the duration of the course, students practiced meditation and mindfulness. In the last week of the course, participants completed the post-test critical thinking instrument. This study was given ethical approval by the University of Phoenix Institutional Review Board.

\section{Study design}

The study employed a quantitative, causal comparative research design to examine the relationship of the unknown effects of individual characteristics related to age, ethnicity, gender and the improvement of critical thinking skills using meditation. Descriptive statistics are appropriate analyzes when the goal of the research is to present the participant's responses to survey items to address the research questions. Both descriptive and inferential statistics were used to describe demographics. Inferential statistics are conducted when the goal of the research is to draw conclusions about the statistical significance of the relationships and/or differences among variables of interest [32]. For this study, both the Wilcoxon Mann-Whitney Test and the Kruskal-Wallis Test were conducted on the measures of critical thinking after engaging in meditation and mindfulness.

\section{Apparatus and materials}

All computer tasks were conducted on a Hewlett-Packard (HP) computer with a 15 in LCD Monitor. The Critical Thinking Instrument was administered through Insight Assessment's secured, encrypted online testing system. The Mindfulness Survey was administered through Survey Monkey.

\section{Mindful attention awareness scale (MAAS) survey}

The trait MAAS is a 15-item scale designed to assess a core characteristic of mindfulness, which focused on the receptive state of mind in which attention is informed by a sensitive awareness of what is occurring in the present and focused on observing what is taking place. The MAAS employs a sixpoint Likert scale (e.g. $1=$ almost always, $2=$ very frequently, $3=$ somewhat frequently, $4=$ somewhat infrequently, $5=$ very infrequently, and $6=$ almost never). Factor analyses with undergraduate, community, and nationally sampled adult and adult cancer populations have confirmed a single factor scale structure. Internal consistency (Cronbach's alphas) generally ranges from 0.80 to 0.90 . The MAAS also has demonstrated high test-retest reliability, discriminant and convergent validity, known groups validity, and criterion validity $[33,34]$.

\section{California critical thinking skills test plus numeracy (CCTST-N)}

The CCTST-N is an objective measure of the core 
reasoning skills needed for reflective decision making that also includes measuring numeracy in addition to critical thinking skills. Multiple choice items on the CCTST-N use everyday scenarios that range in difficulty and complexity and this assessment is typically administered in 45-50 minutes. For this study, the CCTST-N was administered to all participants to measure eight critical thinking skills, including mathematical reasoning skills. These skills included the following: (a) Analysis; (b) Interpretation; (c) Inference; (d) Evaluation; (e) Explanation; (f) Induction; (g) Deduction; (h) Overall Reasoning Skills; and (i) Numeracy. The appropriate internal consistency reliability for Insight Assessment Critical Thinking Tests is the KR-20 coefficient. Reliability coefficients range between 0.77-0.83 [35].

\section{Procedure}

All participants first completed the MAAS and the CCTST-N assessment online at a separate location (e.g. home or campus computers). In the first week of the course, participants consented to participant in the study and completed both the MAAS and Pretest of the CCTST-N. Through the duration of the course, students practiced meditation and mindfulness. In the last week of the course, participants completed the post-test critical thinking instrument.

\section{Findings}

The research questions focused on whether there was a statistical difference between improvement of critical thinking skills and meditation in online higher education according to age, ethnicity and gender. To evaluate these predictions, we conducted both the Wilcoxon Mann-Whitney Test and the Kruskal-Wallis Test examining the differences and all interactions. Results revealed that there is no significant statistical difference based on the effect of age, ethnicity, and gender and the interaction between them.

\section{Sample distribution due to age, ethnicity and gender}

Twenty-two participants completed the survey instrument, 12 participants $(54.5 \%)$ and 10 participants $(45.5 \%)$. The respondents' ages ranged from 20-64, with a mean of 39.6 years. Most respondents were White/Caucasian, Anglo American, (45.5\%), followed by Black, African American (22.7\%), Other (22.7\%), Hispanic, Latino, Mexican American $(4.5 \%)$ and one respondent $(4.5 \%)$ chose not to provide this information. Most of the respondents were female (86.4\%), while the other respondents were male (13.6\%).

\section{Overall critical thinking and its dimension}

In this study, the sample had a moderate level of overall critical thinking with relative weight $(70.7 \%)$ and a mean ( $M=67.89$; SD 5.72). In terms of the dimensions, the highest dimension was Interpretation $(74.9 \%)$, followed by Induction (74.3\%), Explanation (73.2\%), Inference (70.3\%), Evaluation (69.9), Deduction (68.2\%), Numeracy (67.8\%) and Analysis $(67.3 \%)$.

\section{Effect of age on critical thinking skills and meditation}

Our first hypothesis predicted that there was not a statistical difference based on the effect of age on improvement of critical thinking skills as a result of using meditation. To evaluate these predictions, we conducted a Mann-Whitney $\mathrm{U}$ analysis examining the differences according age and the critical thinking dimensions. For an alpha level of 0.05 , the mean ranks between age groups $(68.41, n=10$ for ages 40 years and above; $67.45, \mathrm{n}=12$ for ages 39 years and less) were not significantly different, $z=-0.363, p=0.722$ ). Therefore, it is appropriate to deem that our hypothesis was accepted because data revealed that there was not a statistical difference based on the effect of age on improvement of critical thinking skills as a result of using meditation.

\section{Effect of ethnicity on critical thinking skills and meditation}

Our second hypothesis predicted that there was not a statistical difference based on the effect of ethnicity on improvement of critical thinking skills as a result of using meditation. To evaluate these predictions, we conducted a Mann-Whitney $U$ analysis examining the differences according ethnicity and the critical thinking dimensions. For an alpha level of 0.05 , the mean ranks between ethnic groups (67.87, n=10 for White, Caucasian, Anglo American; 67.58, $\mathrm{n}=5$ for Black, African American; 62.64, $\mathrm{n}=1$ for Hispanic, Latino, Mexican American; 71.04, n=5 for other; 59.04, $n=1$ for participants who chose not to provide this information) were not significantly different, $z=5.479, \quad p=0.242$ ). Therefore, it is appropriate to deem that our hypothesis was accepted because data revealed that there was not a statistical difference based on the effect of ethnicity on improvement of critical thinking skills as a result of using meditation.

\section{Effect of gender on critical thinking skills and meditation}

Our third hypothesis predicted that there was not a statistical difference based on the effect of gender on improvement of critical thinking skills as a result of using meditation. To evaluate these predictions, we conducted a Mann-Whitney U analysis examining the differences according gender and the critical thinking dimensions. For an alpha level of 0.05 , the mean ranks between gender $(68.40, \mathrm{n}=3$ for male participants; $67.45, n=12$ for female participants) were not significantly different, $z=-0.191, p=0.857)$. Therefore, it is appropriate to deem that our hypothesis was accepted because data revealed that there was not a statistical difference based on the effect of gender on improvement of critical thinking skills as a result of using meditation.

\section{Discussion and Conclusion}

In the online classroom experience, the goal was for students to learn about meditation and mindfulness practices. This study focused to quantify the strength of relationships across age, gender and ethnicity to the use of meditation to improve critical thinking. Based on the limited research that has been conducted on these relationships, the researchers 
hypothesized that a significant statistical difference will not exist based on the effect of age, gender and ethnicity on improvement of critical thinking skills using meditation. The lack of significance in this finding may contribute to the lack of power in the analysis due to a smaller sample size. It is important to note that the number of weeks of the two courses differed by one week as the study site added a new version of the same course. Additionally, the course allowed for students to engage in meditation for a minimum of 5-10 min daily throughout the duration of the meditation courses. There were some students who did engage in the minimum meditation requirements, while there were other students who expanded their daily meditation in increased varying amounts of time. Helber et al. [21] found that students need to meditate for a certain amount of time before any real changes in cognition can be experienced. From the data findings in this study, statistical evidence indicates that age, ethnicity, and gender are not significant factors in the improvement of critical thinking skills and meditation in online higher education.

There were several limitations for this study. The first limitation was the lack of the critical thinking instrument and survey completion. Based on a lack of survey completion by a wide range of students, the generalization of the study is limited. If a larger number of students had completed the study, a significant statistical difference may have existed between or among groups. The predominate gender group was female. If more males had responded to individual survey questions, a change in the significant distribution of percentages of Almost Always, Very Frequently, Somewhat Frequently and Somewhat Infrequently and Very Infrequently responses may have been noted. If more non-White, Caucasian or Anglos had participated, the ethnicity question responses percentages could have affected the significance level of the findings. The second limitation was the nature of the questions asked in the critical thinking instrument and survey. Over 60 students were invited to participate in the survey and the nature of the questions asked could have been a reason for a lower response rate than anticipated. Students who received the survey may not have understood how the questions related to meditation and mindfulness. The third limitation for this study might have been that students with full schedules lacked time to complete the survey when it was sent to them. The fourth limitation is that some of the meditation and mindfulness courses had low enrollment and had to be cancelled resulting in no surveys being conducted.

The findings and limitations of this study indicate a number of potential avenues for future research. It would be valuable to conduct a further study with a larger sample size, comparing the experiences and perceptions of meditation and mindfulness in both virtual and face-to-face higher education courses. Specifically, online higher education leaders may want to use the variables in this study to explore female and male students' perceptions on the role of meditation and mindfulness and the application of critical thinking skills in the online classroom. Additionally, online higher education leaders may benefit from the exploration of age students' perceptions based on age and ethnicity.

\section{References}

1. Waters L, Barsky A, Ridd A, et al. Contemplative education: A systematic, evidence-based review of the effect of meditation interventions in schools. Educ Psychol Rev. 2015;27(1):103-34.

2. Sirois FM, Tosti N. Lost in the moment? An investigation of procrastination, mindfulness and well-being. J Ration Emot Cogn Behavr Ther. 2012;30:237-48.

3. Evans DR, Baer R, Segerstrom SC. The effects of mindfulness and self-consciousness on persistence. Pers Individ Diff. 2009;47:379-82.

4. Ostafin BD, Bauer C, Myxter P. Mindfulness decouples the relation between automatic alcohol motivation and heavy drinking. J Soc Clin Psychol. 2012;31:729-45.

5. Jordan $\mathrm{CH}$, Wang $\mathrm{W}$, Donatoni L, et al. Mindful eating: Trait and state mindfulness predict healthier eating behavior. Pers Individ Diff. 2014;68:107-11.

6. Haynes DJ, Irvine K, Bridges M. The blue pearl: The efficacy of teaching mindfulness practices to college students. Buddhist-Christian Studies. 2013;33:63-82.

7. Ritchhart R, Perkins DN. Life in the mindful classroom: Nurturing the disposition of mindfulness. J Soc Issues. 2000;56(1):27.

8. O'Donnell A. Contemplative pedagogy and mindfulness: Developing creative attention in an age of distraction. J Philos Educ. 2015;49(2):187-202.

9. Bellin ZJ. The meaning connection between mindfulness and happiness. Journal of Humanistic Counseling. 2015;54:221-35.

10. Colzato L, Ozturk A, Hommel B. Meditate to create: The impact of focused-attention and open-monitoring training on convergent and divergent thinking. Front Psychol. 2012;3:116.

11. Ching H, Koo M, Tsung-Huang T, et al. Effects of mindfulness meditation course on learning and cognitive performance among university students in Taiwan. Evid Based Complement Alternat Med. 2015.

12. Noone C, Bunting B, Hogan MJ. Does mindfulness enhance critical thinking? Evidence for the mediating effects of executive functioning in the relationship between mindfulness and critical thinking. Front Psychol. 2016;1-16.

13. Mrazek M, Franklin M, Phillips D, et al. Mindfulness training improves working memory capacity and GRE performance while reducing mind wandering. Psychol Sci. 2013;24(5):776-81.

14. Burton CL, Strauss E, Hultsch DF, et al. Cognitive functioning and everyday problem solving in older adults. Clin Neuropsychol. 2006;20:432-52. 
15. Webster-Wright A. The eye of the storm: A mindful inquiry into reflective practices in higher education. Reflective Practice. 2013;14(4):556-67.

16. Dunn R, Callahan JL, Swift JL. Psychotherapy. American Psychological Association. 2013;50(3):312-5.

17. Kiener M, Ahuna KH, Tinnesz CG. Documenting critical thinking in a capstone course: Moving students toward a professional disposition. Educational Action Research. 2014;22(1):109-21.

18. Leppma M. Loving-kindness meditation and counseling. J Ment Health Couns. 2012;34(3):197-204.

19. Fulton CL, Cashwell CS. Mindfulness-based awareness and compassion: Predictors of counselor empathy and anxiety. Counselor Education Supervision. 2015;54(2):122-33.

20. Shapiro S, Lyons K, Miller R, et al. Contemplation in the classroom: A new direction for improving childhood education. Educ Psychol Rev. 2015;27(1):1-30.

21. Helber C, Zook N, Immergut M. Meditation in higher education: Does it enhance cognition? Innovative Higher Education. 2012;37(5):349-58.

22. Oaklander M. The mindful classroom. Time 2016;188(13):44-7.

23. Black D, Fernando R. Mindfulness training and classroom behavior among lower-income and ethnic minority elementary school children. J Child Fam Stud. 2014;23(7):1242-6.

24. Viafora D, Mathiesen S, Unsworth S. Teaching mindfulness to middle school students and homeless youth in school classrooms. J Child Fam Stud. 2015;24(5):1179-91.

25. Wisner BL, Jones B, Gwin D. School-based meditation practices for adolescents: A resource for strengthening self-regulation, emotional coping and self-esteem. Child Sch. 2010;32(3):150-9.
26. Byrne C, Bond LA, London M. Effects of mindfulnessbased versus interpersonal process group intervention on psychological well-being with a clinical university population. Journal of College Counseling. 2013;16(3):213-27.

27. Hanley A, Warner A, Garland E. Associations between mindfulness, psychological well-being and subjective well-being with respect to contemplative practice. J Happiness Stud. 2015;16(6):1423-36.

28. Paul R, Elder L. The miniature guide to critical thinking concepts and tools. Foundation for Critical Thinking Press, Dillon Beach. 2010.

29. Sergeant AA, Camion DM. Critical thinking development through teaching: A sample project in accounting. Business Education Innovation Journal. 2016;8(2):18897.

30. Chiesa A, Calati R, Serretti A. Does mindfulness training improve cognitive abilities? A systematic review of neuropsychological findings. Clin Psychol Rev. 2011;31:449-64.

31. Short MM, Mazmanian D, Oinonen K, et al. Executive function and self-regulation mediate dispositional mindfulness and well-being. Pers Individ Diff. 2015.

32. Statistics Solution. When to use descriptive statistics to answer RQs. 2017.

33. Brown KW, Ryan RM. The benefits of being present: Mindfulness and its role in psychological well-being. J Pers Soc Psychol. 2003;84:822-48.

34. Carlson LE, Brown KW. Validation of the mindful attention awareness scale in a cancer population. J Psychosom Res. 2005;58:29-33.

35. https://www.insightassessment.com/Products/ProductsSummary/Critical-Thinking-Skills-Tests/CaliforniaCritical-Thinking-Skills-Test-Numeracy-CCTST-N

\section{*Correspondence to:}

Alicia Holland

School of Advanced Studies

University of Phoenix

PO Box 94224, Phoenix

Arizona, 85070

USA

Tel: 480-788-7374

Fax: 512-233-5389

E-mail: drhollandj@gmail.com;

drhollandj@email.phoenix.edu 